\title{
Particles: A New International Open Access Journal for Nuclear and Particle Physics
}

\author{
Roy A. Lacey ${ }^{1,2}$ \\ 1 Department of Chemistry, Stony Brook University, Stony Brook, New York, NY 11794-3400, USA; \\ roy.lacey@stonybrook.edu \\ 2 Department of Physics, Stony Brook University, Stony Brook, New York, NY 11794-3800, USA
}

Received: 6 November 2017; Accepted: 6 November 2017; Published: 9 November 2017

Particles, a new online, open access journal, has been launched to facilitate the publication of original research papers, review articles, and communications of new results and progress in a broad set of subfields which span nuclear and particle physics, experimental/theoretical high-energy physics and astrophysics. A primary objective of Particles is to encourage, facilitate and disseminate detailed accounts of experimental and theoretical findings by scientists across the globe. By design, the journal is staffed with a first-rate editorial board supporting a broad scope of topics:

- Nuclear structure, reactions, and dynamics

- Nuclear forces and few-body systems

- Rare decays and fundamental symmetries

- Nuclear astrophysics

- Intermediate and high energy heavy ion physics

- Condensed matter theories in particle physics

- Experimental data processing

- Particle detection

- Particle accelerators

- Novel hardware solutions

- Lattice field theory

- Hadronic physics and QCD

- High-energy particle physics

- Neutrino physics

- Astroparticle physics

- Particle physics in cosmology

- Quantum field theory methods in particle physics

- Statistical physics of elementary particles

Thus, Particles also offers an excellent opportunity to publish the latest multi-disciplinary results, with a high potential to reach a much broader scientific community, stakeholders, and the interested layperson.

High quality, innovative, pioneering and relevant manuscripts are currently being invited. As outlined on the journal's website, these submissions will be subject to a rigorous peer review process and, if accepted, published online, providing as short a turnaround time as possible. The Editorial Board also recognizes the value of in-depth coverage. Therefore, no restrictions are placed on the length of manuscript submissions. The latter provides an important opportunity to share data and results in detail. 
MDPI, with 21 years of publishing experience, boasts a professional editorial staff currently publishing more than 186 peer-reviewed journals, of which all major journals are already covered by the leading databases, such as Science Citation Index (Web of Science), Scopus, PubMed, EI etc. Long-term archiving is guaranteed through the immediate deposit of published articles with the Swiss National Library (SNL).

The Editorial Board sees Particles as an exciting new journal, and we are fully committed to its success, which will be characterized by its high quality and cutting-edge research. We believe that your contributions can help us to achieve this goal while we disseminate the results of your work to the broader scientific community.

On behalf of the Editorial Board, I invite you to submit your manuscripts, review papers and suggestions for Special Issues to Particles, and I look forward to receiving your contributions.

(C) 2017 by the author. Licensee MDPI, Basel, Switzerland. This article is an open access article distributed under the terms and conditions of the Creative Commons Attribution (CC BY) license (http://creativecommons.org/licenses/by/4.0/). 